\title{
Weakness after foreign travel in a 40-year-old man
}

\author{
G. C. Colleran • M. J. Shelly • M. R. Moynagh • \\ E. C. Kavanagh
}

Published online: 4 March 2010

(C) ISS 2010

Keywords MRI · Gadolinium · Weakness

\section{Diagnosis}

Acute inflammatory demyelinating polyneuropathy (GuillainBarré syndrome) following hepatitis E infection.

\section{Discussion}

The diagnosis of hepatitis E was made from abnormal liver function tests, a recent history of foreign travel and serology (anti-HEV IgM antibodies identified in serum) consistent with acute hepatitis $\mathrm{E}$ virus (HEV) infection and could not be verified by imaging.

Guillain-Barré syndrome is an acute inflammatory demyelinating polyneuropathy [1-4] first described in 1916 by three French neurologists: Georges Guillain, Jean-Alexandre Barré and André Stohl [3]. There are at least four described subtypes, the most common of which is AIDP: acute inflammatory demyelinating polyradiculoneuropathy [3]. The clinical diagnosis is usually established based on clinical criteria, electrophysiological studies and CSF analysis [1]. AIDP is an auto-immune disease

The case presentation can be found at doi:10.1007/s00256-010-0905-5

G. C. Colleran $(\varangle) \cdot$ M. J. Shelly $\cdot$ M. R. Moynagh •

E. C. Kavanagh

Department of Radiology,

Mater Misericordiae University Hospital,

Eccles Street,

Dublin 7, Ireland

e-mail: collerangabrielle@hotmail.com postulated to be triggered by a preceding viral or bacterial infection, the most common of which are thought to be Campylobacter jejuni, Epstein-Barr virus (EBV), Mycoplasma pneumoniae and cytomegalovirus (CMV) [5].

Magnetic resonance imaging of the lumbar spine in this case demonstrated diffusely abnormal homogeneous low T1 signal intensity of vertebrae throughout the spine on sagittal T1-weighted imaging, which was related to anaemia. Non-contrast-enhanced imaging revealed no discrete cord abnormality or plaque (see Fig. 1a). Following contrast medium administration there was diffuse enhancement of the conus and cauda equina with enhancement and thickening of the filum terminale (see Fig. 1b).

The cauda equina enhances after gadolinium in patients with Guillain-Barré syndrome (GBS) [1] because of a breakdown of the blood-nerve barrier that should be present [4].

Gorson et al. in a prospective evaluation of MRI findings in patients with GBS concluded that prominent enhancement of the cauda equina was $83 \%$ sensitive for GBS, present in $95 \%$ of typical presentations and was associated with an increased GBS disability score [1]. Byun et al. in a retrospective review of MRI findings in patients with GBS concluded that while enhancement of the intrathecal spinal nerve roots is not specific to GBS, enhancement of the anterior spinal nerve roots in isolation is highly suggestive of GBS [4].

Guillain-Barré syndrome has been described is post hepatitis $\mathrm{E}$ infection and it is postulated that this association might be due to molecular mimicry [5]. Molecular mimicry describes the situation where the host mounts an immune response to the infectious organism, in this case HEV, because HEV has epitopes homologous to the host's own peripheral nerves [5]. 
It is proposed that in patients with GBS induced by HEV infection, the HEV-infected cells express an epitope (proposed to be a glycolipid), which induces an anti-ganglioside response, resulting ultimately in axonal degeneration [5].

Enhancement of the cauda equina is not specific to GBS. The differential diagnosis includes neoplasia, tuberculosis, neurobrucellosis, CMV radiculitis, disc herniation with associated root inflammation, neurofibromata, herpes zoster infection, Dejerine-Sottas syndrome, Charcot-Marie-Tooth (CMT) syndrome, schwannoma, Lyme disease, arachnoiditis, granulomatous diseases including sarcoid and other inflammatory processes $[2,6]$. With tuberculous arachnoiditis leptomeningeal enhancement may be associated with enhancing nodules [6].

With respect to immunocompromised patients, in particular those with acquired immune deficiency syndrome (AIDS), non-specific enhancement of nerve roots is demonstrated in AIDS-related polyradiculopathy in association with metastatic disease, arachoiditis (which can occur after infection, surgery and subarachnoid haemorrhage) was previously common after contrast myelography, but has decreased in incidence since the introduction of non-ionic contrast media [6], sarcoidosis and CMV infections [4]. CMV causing AIDS-related polyradiculopathy causes diffuse enhancement of the cauda equina in a manner similar to that of GBS [4].

\section{References}

1. Gorson KC, et al. Prospective evaluation of MRI lumbosacral nerve root enhancement in acute Guillain-Barre syndrome. Neurology. 1996;47(3):813-7.

2. Berciano J, Pascual J. Selective contrast enhancement of anterior spinal nerve roots on magnetic resonance imaging: a suggestive sign of Guillain-Barre syndrome and neurobrucellosis. J Peripher Nerv Syst. 2003;8(3):135.

3. Hughes RA, Cornblath DR. Guillain-Barre syndrome. Lancet. 2005;366(9497):1653-66.

4. Byun WM, et al. Guillain-Barre syndrome: MR imaging findings of the spine in eight patients. Radiology. 1998;208 (1):137-41.

5. Loly JP, et al. Guillain-Barre syndrome following hepatitis E. World J Gastroenterol. 2009;15(13):1645-7.

6. Grossman RI, Yousem DM. Neuroradiology: the requisites. 2nd ed. St. Louis: Mosby; 2003. 